\title{
Esquisse sur la situation actuelle des relations entre sciences sociales et dispositif développeur en Allemagne
}

\section{Gudrun Lachenmann}

\author{
(2) OpenEdition \\ Journals \\ Édition électronique \\ URL : http://journals.openedition.org/apad/303 \\ DOI : 10.4000/apad.303 \\ ISSN : 1950-6929 \\ Éditeur \\ LIT Verlag \\ Édition imprimée \\ Date de publication : 15 mars 1991 \\ Référence électronique \\ Gudrun Lachenmann, «Esquisse sur la situation actuelle des relations entre sciences sociales et \\ dispositif développeur en Allemagne », Bulletin de l'APAD [En ligne], 1 | 1991, mis en ligne le 23 juin \\ 2006, consulté le 08 septembre 2020. URL : http://journals.openedition.org/apad/303 ; DOI : https:// \\ doi.org/10.4000/apad.303
}

Ce document a été généré automatiquement le 8 septembre 2020.

Bulletin de I'APAD 


\title{
Esquisse sur la situation actuelle des relations entre sciences sociales et dispositif développeur en Allemagne
}

\author{
Gudrun Lachenmann
}

1 La situation peut être caractérisée par quelques contradictions entre les discours et les pratiques institutionnelles, qui peuvent être résumées comme suit :

2 Le dispositif développeur, depuis quelques années, exprime son insatisfaction devant la contribution, selon lui, déficiente et peu constructive, des sciences sociales à la "dimension socio-culturelle du développement" ${ }^{1}$, telle qu'elle a été "découverte" il y a quelques années par les développeurs. Un groupe de travail comprenant chercheurs et institutions n'ayant pas eu de suite, c'est seulement récemment que l'agence technique allemande de coopération (Deutsche Gesellschaft für technische Zusammenarbeit GTZ, Eschborn/Frankfurt) a relancé l'initiative en organisant un séminaire cette fois non sur le thème comme tel mais sur les formes possibles de coopération chercheurs/ institutions - en invitant quelques représentants d'autres pays d'Europe mais sans résultats très concrets.

3 Cependant, les agences exécutives de coopération technique (GTZ) et financière (Kreditanstalt für Wiederaufbau Kfw, Frankfurt) seront bien obligées d'agir, étant donné que le Ministère de la Coopération Economique (Bundesministerium für wirtschaftliche Zusammenarbeit BMZ, Bonn) a récemment officialisé le principe de tenir compte de la "dimension socio-culturelle" dans toutes les étapes de la coopération technique. Cette conception est basée sur trois "facteurs-clés" "légitimité, état du développement et hétérogénéité".

4 Dans le débat précédent, les chercheurs ont critiqué cette conception sur plusieurs aspects. Entre autres, ils ont formulé le reproche qu'après avoir été tout-à-fait technocratique, la coopération évitant (apparemment) toute "intervention dans la société", elle évite à présent d'aborder les questions de structure sociale et de pouvoir avec son renforcement par la coopération etc.) en se lançant sur le thème en apparence 
neutre de la culture avec un fort penchant pour le "mythe du traditionalisme" (Elwert) 2 .

5 L'ancienne conception de la société qui est à la base du "paradigme développeur" est encore une fois évidente. Il s'agit de l'idée mécaniste selon laquelle on peut transformer la société à volonté (pourvu qu'on connaisse les recettes). Ainsi, les représentants des sciences sociales reprochent aux décideurs politiques de ne considérer que le socio-technique, évacuant ainsi la dimension critique des sciences sociales, le caractère subjectif de la société et de la culture, et l'existence des structures de pouvoirs.

6 D'un côté, la coopération allemande adhère absolument au credo de la Banque Mondiale et $\mathrm{du}$ Fonds Monétaire International concernant la philosophie du développement et, très concrètement, la théorie économique nourrissant l'ajustement structurel. On poursuit un libéralisme pur en oubliant presque le modèle d'économie sociale de marché créé en Allemagne. On parle certes beaucoup de la dimension sociale de l'ajustement en la réduisant aux questions d'assistance sociale.

7 D'un autre côté, on croit beaucoup aux "conditionnalités". Auparavant on parlait de "dialogue politique", des "conditions-cadre" (la Banque Mondiale utilise depuis peu le concept de "enabling environment") comme si le Gouvernement pouvait changer la société à son gré en affrontant quelques problèmes (tels que le planning familiale, la promotion féminine) avec sa seule "volonté politique".

8 En évitant de discuter sur les implications et conditions de pouvoir et de structure sociale en vue d'homogénéiser les structures non seulement économiques mais également sociales, on exige la démocratisation (concernant surtout le système conventionnel de démocratie parlementaire) et le respect des droits de l'homme, sans analyser les processus et forces nécessaires en tant qu' agents d'une société civile.

9 En même temps, sans voir la contradiction envers les mesures macro-économiques, on parle beaucoup d'auto-promotion, de lutte contre la pauvreté, etc...

10 Tout cela montre que le discours officiel sur le développement a complètement changé depuis quelques années, bien que la séance annuelle du FMI et de la BM à Berlin en 1988 ait suscité un débat public d'une envergure inattendue. Le discours académique et politique avait été caractérisé par un tiermondisme de gauche, dans la tradition de l'économie politique et du structuralisme, avec très peu d'aspects sociologiques et anthropologiques. D'un côté, l'ethnologie était discréditée comme "science coloniale" ; de l'autre et c'est encore vrai dans beaucoup de cas aujourd'hui - elle évitait tout contact avec le dispositif développeur porteur de normativité et de transfert culturel. Cela est probablement une des raisons pour lesquelles il y a si peu d'interactions fructueuses entre développement et sciences sociales.

11 Contrairement au libéralisme progagé et au désengagement de l'État exigé partout, la coopération élabore des méthodes de plus en plus raffinées de planification, telle que la méthode de la "planification orientée vers les objectifs" (zielorientierte Projektplanung)- basée sur d'anciennes conceptions utilisées aux États Unis pour la planification sociale et la recherche d'évaluation. En principe, on prétend disposer ainsi d'un cadre heuristique ouvert à participation de tous les agents et à la prise en considération de tous les aspects; en réalité cela introduit une rigidité accrue. Simultanément, on considère que l'on peut inclure la dimension socio-culturelle dans les différentes étapes de planification au moyen d'indicateurs et de "check-lists", à la 
manière d'une procédure administrative et sans que pour autant les représentants des sciences sociales interviennent. Ceux-ci, souvent, ne sont autorisés qu'à écrire le dernier chapitre d'un rapport, sur les "traditions" d'une société etc., sans liaison avec le reste des concepts et mesures envisagées. Aussi parle-t-on beaucoup de "groupes-cibles" et d'objets d'intervention en "compatibilité sociale" (Sozialvertrâgl ichkeit).

La reconnaissance récente de la "dimension socioculturelle" consiste en une plus grande concession de la part des planificateurs à l'autonomie des "bénéficiaires" afin qu'eux-mêmes puissent agir selon leurs propres logiques. Mais cette prise de conscience est lente et il est absolument nécessaire de stimuler les sciences sociales dans les pays mêmes et de promouvoir leur inclusion dans la coopération, au lieu de trouver des postes pour des représentants des sciences sociales dans nos pays affectés par le chômage.

Bien sûr, ils sont nécessaires des deux côtés afin d'assurer une communication inter-culturelle adéquate. C'est seulement récemment qu'on commence à engager des bureaux d'études locaux et même à installer un nouveau programme d'appui universitaire (en Afrique de l'Est) - après une longue abstention. Le problème est, cependant, qu'il n'existe pas un discours public sur les implications du développement, inspiré ou guidé par les sciences sociales dans les pays du tiers-monde pour des raisons politiques et autres. Par exemple ces bureaux d'études ne sont pas tenus de respecter les règles académiques au contraire, leurs travaux ne doivent pas être publiés (le même problème de confidentialité existe chez nous concernant les universitaires travaillant pour la coopération).

En ce qui concerne le manque d'orientation sociologique et anthropologique du discours de la théorie du développement, il y a eu des améliorations assez importantes, et différentes approches nouvelles ont été formulées dans différentes universités (Bielefeld, Berlin, etc.). La section de sociologie du développement de la société allemande de sociologie s'est intitulée "anthropologie sociale et sociologie du développement" il y a déjà quelques années. Mais ce n'est que très récemment que la Société Allemande d'Ethnologie a constitué un groupe de travail sur "l'ethnologie du développement".

Cependant, les universités semblent - par malentendu - considérer la théorie sociologique du développement comme étant trop normative, et il y a une certaine tendance à supprimer les chaires et postes en faveur d'une sociologie culturelle récemment revitalisée.

16 Pourtant il existe encore des possibilités de valoriser la sociologie et l'anthropologie dans la discussion académique générale. Il est frappant que l'anthropologie internationale du développement intègre depuis peu des débats qui se sont développés en Allemagne tels que les approches en termes de théorie de l'action, d'ethnométhodologie, de paradigme interprétatif, de phénoménologie, de sociologie du savoir telle que la "construction sociale de la réalité" (Berger/Luckmann) etc.

17 En ce qui concerne l'institutionnalisation des études sur le développement. quelques universités offrent la possibilité de les choisir comme sujets principaux pour la maitrise, le diplôme ou le doctorat. Il y a quelques cours post-gradués d'études de développement, tels que les études rurales à l'université de Goettingen, la sociologie du développement à Bielefeld, ou le "master" en "infra structural planning" (Stuttgart, Dortmund) et "community health" (Heidelberg), destinés surtout aux personnes venant 
du tiers-monde et ayant une certaine expérience professionnelle. Hors de l'université, l'institut allemand de développement (avec un budget permanent du Ministère de la coopération) prépare des Allemands comme futurs cadres de la coopération.

\section{NOTES}

1.G. Lachenmann, 1988, Sozio-kulturelle 8edingungen und Wirkungen in der Entwicklungszusemmenarbeit, Berlin : Deutsches Institut für Entwicklungspolitik.

2.Georg Elwert, 1983 "Der entwicklungssoziologische Mythos vom Traditionalismus", in : D. Goetze, H. Weiland. ed., Soziokulturelle Implikationen technologischer Wandlungsprozesse, Saarbrucken : Breitenbach, p.29-55. 